\title{
First Combined Heart-Liver Transplant in Amyloidosis Due to Transthyretin Mutation in Brazil: Impact of the Liver on Reducing anti-HLA Antibodies
}

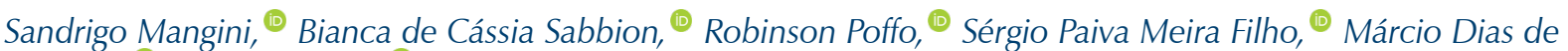 \\ Almeida, ${ }^{(1)}$ Fernando Bacal ${ }^{(0)}$ \\ Hospital Israelita Albert Einstein, São Paulo, SP - Brazil
}

\section{Introduction}

Cardiac amyloidosis is characterized by the presence of deposits of amyloid material between myocardial fibers, leading to impaired function. In $95 \%$ of cases, it can be secondary to excessive production of abnormal light chain immunoglobulins or changes in transthyretin (TTR), either due to mutation or wild type. TTR is a protein produced mainly by the liver, and it is responsible for transporting retinol and thyroid hormone. Familial amyloidotic polyneuropathy (FAP) is an autosomal dominant genetic disease due to mutation in the TTR gene, and it has high morbidity and mortality, especially when there is cardiac involvement. More than 130 mutations have been described, and they determine phenotypes of neurological and/or cardiac predominance. Historically, liver transplantation was the standard treatment to reduce progression of FAP; in the presence of heart disease with advanced heart failure (HF), heart transplantation may be considered. Combined heart-liver transplant (CHLT), although uncommon, is a well-established indication for transthyretin amyloidosis (ATTR), with good outcomes; furthermore, the presence of the liver seems to minimize the risks of cellular or antibody-mediated rejection, which could be of interest for patients with high immunological risks, especially those with human leukocyte antigen (HLA) hypersensitivity.

\section{Objective}

To report the first CHLT in Brazil, performed in a patient with ATTR with a mixed phenotype, in the context of cardiogenic shock, who, as an additional risk factor, had HLA hypersensitivity.

\section{Case Report}

"RGP," a 50-year-old female patient, who worked as a housewife and had 2 children, began to have a condition

\section{Keywords}

Amyloidosis; Double heart-liver transplant

Mailing Address: Sandrigo Mangini •

Rua Lúcio Martins Rodrigues, 330, ap 13. Postal Code:05621-025, Jardim Leonor, São Paulo, SP - Brazil.

E-mail: sandrigomangini@cardiol.br, sandrigoman@uol.com.br Manuscript received September 27, 2021, revised manuscript October 12 2021, accepted October 12, 2021.

DOI: https://doi.org/10.36660/abchf.20210029 of HF associated with sensory motor polyneuropathy, orthostatic hypotension, and diarrhea, in 2013; regarding family history, she reported that her father and brother had died of undefined heart disease at the age of 50; in 2015, she had signs and symptoms of HF requiring frequent hospitalizations. Electrocardiogram revealed an anteroseptal electrically inactive area and low voltage in the frontal plane; echocardiogram revealed thickening of the septum and posterior wall of $13 \mathrm{~mm}$, left ventricular diameters $54 \times 43 \mathrm{~mm}$, left ventricular ejection fraction $32 \%$, and severe diastolic dysfunction (restrictive pattern). Magnetic resonance imaging revealed diffuse late enhancement with a non-coronary pattern, suggesting deposition disease. In this context, she underwent endomyocardial biopsy, which was positive for Congo red, defining diagnosis of cardiac amyloidosis. Electroneuromyography showed sensory-motor pattern of upper and lower limbs. She was referred to the liver transplant unit at the Hospital Israelita Albert Einstein, and light chain amyloidosis was ruled out (free light chain, negative serum and urinary immunofixation); heterozygous Glu89Lys mutation in the TTR gene was confirmed, and an evaluation of the heart transplant group was requested. Due to the recurrent hospitalizations due to decompensated HF and difficult clinical management, pre-transplant evaluation tests were requested, including immunological evaluation through the collection of lymphocyte panel reactive antibody (PRA), and the presence of anti-HLA antibodies was identified in $51 \%$ of class I and was negative in class II.

The patient was hospitalized in cardiogenic shock and maintained compensated with inotropic agents and intravenous diuretics; 3 months after admission, on October 17, 2017, she underwent simultaneous CHLT, as a priority recipient, due to inotropic dependence; there was positive virtual crossmatch (donor HLA A 11.23, B 07.35, DR 03.04 and presence of antiB35-MFI 2094) and real crossmatch due to negative complement-dependent cytotoxicity. She received induction with basiliximab. Collection of PRA in the pre-reperfusion portal vein and the post-reperfusion vena cava demonstrated an immediate antibody-reducing effect after the passage of blood through the transplanted liver. The explanted heart and liver were Congo red positive. Time zero liver biopsy showed grade 1 preservation-reperfusion injury. Maintenance immunosuppression was initiated with corticosteroid, tacrolimus, and mycophenolate. On postoperative day 3 after the transplant, she presented an episode of severe abdominal pain, characterized by acute abdomen secondary to extensive intramural hematoma of the ascending colon, and she underwent right hemicolectomy 
and colostomy. She required hemodialysis due to acute renal failure (urea $104 \mathrm{mg} / \mathrm{dl}$ and creatinine $1.4 \mathrm{mg} / \mathrm{dl}$ the day before the transplant) and remained dependent on vasopressor drugs for a prolonged period, requiring the association of fludrocortisone and venlafaxine for weaning from noradrenaline. Due to intense diarrhea and abdominal pain, she did not tolerate the use of mycophenolate, and immunosuppression was maintained with corticosteroids and tacrolimus. Serial endomyocardial biopsies showed no cell rejection $(\mathrm{OR})$; moreover, there were no histological or immunohistological signs (c4d and CD68) of antibodymediated rejection (pAMR0). She showed good hepatic progression, in relation to function and integrity, without requiring biopsy. She was discharged on January 11 , 2018, on postoperative day 86, receiving tacrolimus and prednisone, on intermittent hemodialysis.

The patient underwent intestinal transit reconstruction 6 months after hemicolectomy (April 2018). On account of recurrent episodes of urinary tract infection, in addition to the context of dysautonomia and risk of renal graft loss due to hypotension, kidney transplantation was not indicated. Serially collected PRA demonstrated a fluctuation in the levels of anti-HLA antibodies; however, they were always negative to the specific donor antibody (B35) (Table 1). Endomyocardial biopsies did not show rejection (Table 2), and non-invasive coronary disease evaluations were negative. Over time, there were episodes of hospitalization due to long-term hemodialysis catheter infection and, in the last year, need for surgery to treat a tibial fracture due to a fall. The patient was stable from the point of view of symptoms of polyneuropathy. She maintains outpatient follow-up, with no symptoms of HF; cardiac and liver grafts show no signs of dysfunction, and the patient maintains intermittent hemodialysis. One of the children and niece 30 years old have the same mutation; they have incipient polyneuropathy and heart disease, and they began use of tafamidis after diagnosis.

\section{Discussion}

To the best of our knowledge, this is the first CHLT performed in Brazil; in addition to the fact that it was unprecedented in our country, the presence of HLA hypersensitization, where liver transplantation promoted an immediate reduction of antibodies, was a further interesting characteristic.

CHLT may be indicated for patients with heart diseases that lead to cardiac cirrhosis, especially congenital diseases, patients with liver cirrhosis and concomitant heart disease, and patients with FAP and cardiomyopathy. According to the ISHLT registry, CHLT was responsible for less than $1 \%$ of heart transplants performed in 2019, and FAP was the most common cause, accounting for more than half of cases. It may be performed simultaneously (heart transplant immediately followed by liver transplant, from the same donor) or sequentially (heart transplant preceded or followed by liver transplant, from different donors); in cases of FAP, simultaneous transplantation has been more frequent. Another interesting aspect is the possibility of using the liver of a patient with FAP (domino transplant), in spite of the risk of inducing FAP in the recipients.

In the literature and in Brazil, the Val30Met mutation appears to be the most common, and its relationship with

Table 1 - Evolution of lymphocyte panel reactive antibody

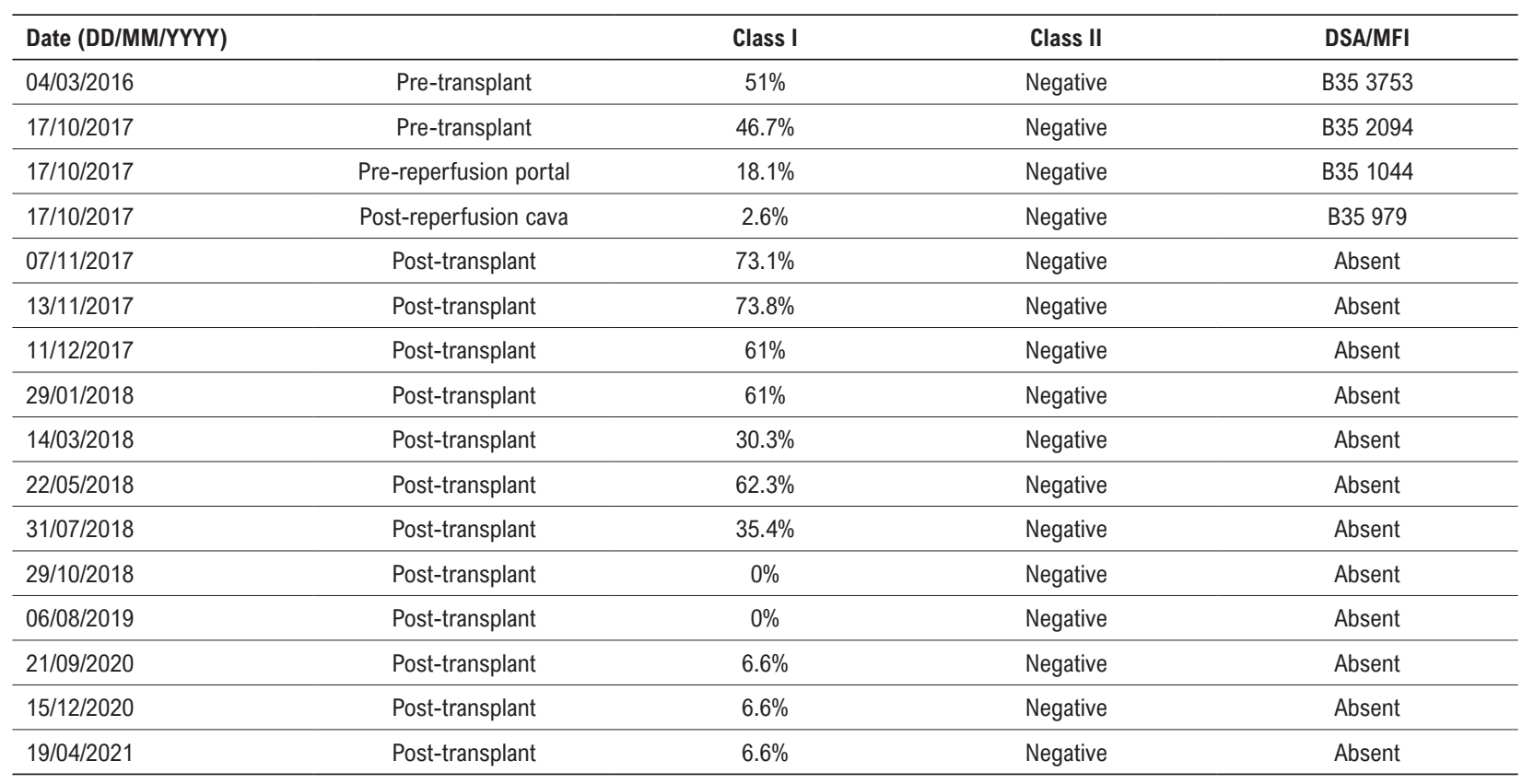

Donor HLA: A 11,23 / B 07,35 / DR 03,04; DSA: donor specific antibody; MFI: median fluorescence intensity. 


\section{Case Report}

Table 2 - Endomyocardial biopsies

\begin{tabular}{lccc}
\hline Date (DD/MM/YYY) & Cellular rejection & Humoral rejection & Congo red \\
\hline $01 / 11 / 2017$ & OR & pAMR 0 & negative \\
\hline $07 / 12 / 2017$ & OR & pAMR 0 & negative \\
\hline $15 / 02 / 2018$ & OR & pAMR 0 & negative \\
\hline $06 / 11 / 2018$ & OR & pAMR 0 & negative \\
\hline $08 / 04 / 2021$ & OR & pAMR 0 & negative \\
\hline
\end{tabular}

pAMR: pathology antibody mediated rejection

the development of advanced cardiomyopathy is less frequent and generally occurs later, with good outcomes by means of isolated liver transplant. On the other hand, the Val122lle mutation, which is present in 4\% of African Americans has shown a predominantly cardiac phenotype, affecting patients over 60 years of age, and it is a differential diagnosis for wild-type ATTR; due to the older age of onset and the inherent risk of combined transplant in this age group, some reports have shown good evolution involving heart transplant alone. Other mutations may have mixed characteristics of involvement, and many of them have already been described in case series, including Glu89Lys, as an indication for CHLT. There appear to be better results with combined transplant compared to isolated liver transplant; however, each case must be evaluated in an individualized manner, considering the mutation and the phenotype/manifestation of the disease at the moment of evaluation. With the development of therapeutic strategies that act in different phases of the evolution of ATTR, it is likely that liver and heart transplants related to FAP become even less frequent; however, in our case, due to advanced cardiomyopathy, including the need for inotropic agents, the indication of simultaneous CHLT was unquestionable. Due to the reduced size of the left ventricular cavity and the frequent involvement of the right ventricle, there may be limitations to the use of long-term mechanical circulatory assist devices. ${ }^{1}$

From an immunological point of view, CHLT shows a lower incidence of rejection, whether cellular or antibody-mediated. ${ }^{3,4}$, Many mechanisms have been proposed to explain the tolerance properties of the liver which may possibly act simultaneously to reduce risk of rejection; among them, we can highlight the ability to secrete soluble class I HLA antigens, allied to the great capacity for absorption and neutralization of alloantibodies directed against HLA antigens. In our case, it was possible to demonstrate the ability to reduce anti-HLA antibodies in the first passage of blood through the transplanted liver; in spite of positive virtual crossmatch, there was a progressive reduction in specific antibody titers against the donor, until negativization; moreover, serial biopsies never demonstrated cellular or antibody-mediated rejection.
Notwithstanding the excellent survival to date, as well as the normal cardiac and liver graft function, multiple complications occurred throughout the reported transplant, including acute abdomen and need for colectomy and transit reconstruction, multiple infections, fracture, and chronic renal failure on dialysis, thus demonstrating the complexity and challenges inherent to CHLT in the population of patients with FAP.

\section{Conclusion}

Patients with FAP and cardiac involvement can benefit from CHLT, as demonstrated in this first Brazilian case report. Notwithstanding the presence of HLA hypersensitivity and specific antibodies against the donor, there was a reduction in titers in the first passage of blood through the liver and the case has progressed without rejection to date, reinforcing the concept of hepatic protection in the context of CHLT.

\section{Author contributions}

Conception and design of the research: Mangini $S$, Sabbion BC, Poffo R, Meira Filho SP, Almeida MD, Bacal F. Writing of the manuscript: Mangini S, Sabbion BC. Critical revision of the manuscript for intellectual content: Mangini S, Sabbion BC, Bacal F.

\section{Potential Conflict of Interest}

No potential conflict of interest relevant to this article was reported.

\section{Sources of Funding}

There were no external funding sources for this study.

\section{Study Association}

This study is not associated with any thesis or dissertation work.

\section{Ethics approval and consent to participate}

This article does not contain any studies with human participants or animals performed by any of the authors. 


\section{References}

1. Simões MV, Fernandes F, Marcondes-Braga FG, Scheinberg P, Correia EB, Rohde LEP, et al. Posicionamento sobre Diagnóstico e Tratamento da Amiloidose Cardíaca - 2021. Arq. Bras. Cardiol. 2021;117(3):561-98. doi: 10.36660/abc. 20210718 .

2. Sousa M, Monohan G, Rajagopalan N, Grigorian A, Guglin M. Heart Transplantation in Cardiac Amyloidosis. Heart Fail Rev. 2017;22(3):317-27. doi: 10.1007/s10741-017-9601-z.

3. Gong T, Hall S. Considerations and Experience Driving Expansion of Combined Heart-Liver Transplantation. Curr Opin Organ Transplant. 2020;25(5):496-500. doi: 10.1097/MOT.0000000000000804.

4. Rizvi SSA, Challapalli J, Maynes EJ, Weber MP, Choi JH, O’Malley TJ, et al. Indications and Outcomes of Combined Heart-Liver Transplant: A Systematic Review and Met-Analysis. Transplant Rev. 2020;34(2):100517. doi: 10.1016/j.trre.2019.100517.

5. Vollmar J, Schmid JC, Hoppe-Lotichius M, Barreiros AP, Azizi M, Emrich T, et al. Progression of transthyretin (TTR) amyloidosis in Donors and Recipients After Domino Liver Transplantation-A Prospective Single-Center Cohort Study. Transpl Int. 2018;31(11):1207-15. doi: 10.1111/tri.13326.
6. Okumura K, Yamashita T, Masuda T, Misumi Y, Ueda A, Ueda M, et al. Long-Term Outcome of Patients with Hereditary Transthyretin V30M Amyloidosis with Polyneuropathy After Liver Transplantation. Amyloid. 2016;23(1):39-45. doi: 10.3109/13506129.2015.1123149.

7. Thenappan T, Fedson S, Rich J, Murks C, Husain A, Pogoriler J, et al. Isolated Heart Transplantation for Familial Transthyretin (TTR) V122I Cardiac Amyloidosis. Amyloid. 2014;21(2):120-3. doi: 10.3109/13506129.2013.853660.

8. Suhr OB, Larsson M, Ericzon BG, Wilczek HE. Survival After Transplantation in Patients with Mutations Other Than Val30Met: Extracts From the FAP World Transplant Registry. Transplantation. 2016;100(2):373-81. doi: 10.1097/TP.0000000000001021.

9. Chou AS, Habertheuer A, Chin AL, Sultan I, Vallabhajosyula P. HeartKidney and Heart-Liver Transplantation Provide Immunoprotection to the Cardiac Allograft. Ann Thorac Surg. 2019;108(2):458-66. doi: 10.1016/j. athoracsur.2019.02.012.

10. Cheng EY. The Role of Humoral Alloreactivity in Liver Transplantation: Lessons Learned and New Perspectives. J Immunol Res. 2017;2017:3234906. doi: $10.1155 / 2017 / 3234906$. 